\title{
About The Edge Covers of the Graph
}

\author{
D. K. Thakkar \\ Department of Mathematics, \\ Saurashtra University, Rajkot, Gujarat, India. \\ B. M. Kakrecha \\ Department of Mathematics, \\ L. E. College, Morbi, Gujarat, India.
}

\begin{abstract}
The paper is about the edge covers of the graph. The change in the edge covering number of the graph is observed under the operations vertex removal, edge removal and edge addition. The necessary and sufficient condition is proved under which the edge covering number decreases when a vertex is removed from the graph also when an edge is added to the graph. The necessary and sufficient condition is proved under which the edge covering number increases when an edge is removed from the graph. Some results about the edge covering number have been obtained by considering the dual hypergraph of the graph under the operation vertex removal from the hypergraph.
\end{abstract}

Keywords: edge cover, edge covering number, hypergraph, dual hypergraph.

AMS subject classification (2010): 05 C 69.

\section{INTRODUCTION}

Various graph operations have been considered in graph theory. In particular, the effect of these operations on the parameters like domination number, independence number, vertex covering number etc. have been studied by several authors. In this paper, we consider three graph operations (namely vertex removal, edge removal and edge addition) and the effect of these operations on the edge covering number of the graph. We prove the necessary and sufficient condition under which the edge covering number decreases when a vertex is removed from the graph. We count the number of minimum edge covers of the graph corresponding to a vertex. We may note that the edge covering number increases or does not change when an edge is removed from the graph also the edge covering number decreases or does not change when a new edge is added between two non-adjacent vertices of the graph. We prove necessary and sufficient condition under which the edge covering number increases when an edge is removed from the graph also the necessary and sufficient condition under which the edge covering number decreases when a new edge is added between two non-adjacent vertices of the graph.

We consider only finite, simple and undirected graphs without isolated vertices. For the graph $G=$ $(V, E)$ with vertex set $V($ or $V(G)$ ) and edge set $E$ (or E $(G)$ ), G - v denotes the subgraph of $G$ obtained by removing a vertex $v$ and all edges incident to $v$. Similarly, for an edge e $\in E, G-e$ is the subgraph of $\mathrm{G}$ obtained by removing an edge e from the graph $\mathrm{G}$. Let $\mathrm{x}$, $\mathrm{y}$ be non-adjacent vertices of the graph $G$ then $G+x y$ is a graph with the same vertex set $V$ of $G$ and with the edge set equal to $E(G) \cup\{x y\}$.

\section{EDGE COVER OF THE GRAPH}

Definition 2.1 [3] A subset $F$ of $E(G)$ is said to be an edge cover of the graph $G$ if every vertex of $G$ is an end vertex of some edge of $F$.

Definition 2.2 An edge cover $F$ of the graph $G$ is said to be a minimal edge cover if for every edge $\mathrm{e} \in \mathrm{F}, \mathrm{F}-\{\mathrm{e}\}$ is not an edge cover of $\mathrm{G}$. 


\section{K. Thakkar \& B. M. Kakrecha}

Theorem 2.3 Let $G$ be a graph and $F$ be an edge cover of $G$. $F$ is a minimal edge cover of the graph $\mathrm{G}$ if and only if for every edge $\mathrm{e}=\mathrm{xy}$ in $\mathrm{F}$, one of the following two conditions hold.

(1) There is no edge (except $\mathrm{e}$ ) incident to $\mathrm{x}$ or every edge incident to $\mathrm{x}$ (except $\mathrm{e}$ ) is outside of $\mathrm{F}$.

(2) There is no edge (except e ) incident to y or every edge incident to y (except e ) is outside of F.

Proof. Suppose that $F$ is a minimal edge cover of $G$ and $e=x y \in F$. Since $F-\{e\}$ is not an edge cover of $G$, there is a vertex $z$ such that $z$ is not covered by any edge of $F-\{e\}$. If $z \neq x$ and $z$ $\neq \mathrm{y}$ then there is an edge $\mathrm{h}$ in $\mathrm{F}$ such that $\mathrm{z} \in \mathrm{h}$ and $\mathrm{h} \neq \mathrm{e}$. Therefore $\mathrm{z} \in\{\mathrm{x}, \mathrm{y}\}$. Suppose that $\mathrm{z}$ $=\mathrm{x}$. Now $\mathrm{x}$ is an end vertex of e but $\mathrm{x}$ is not an end vertex of any edge of $\mathrm{F}-\{\mathrm{e}\}$. Therefore (1) holds. If $\mathrm{z}=\mathrm{y}$ then (2) holds.

Conversely, Suppose that one of the two conditions holds then it is obvious that $\mathrm{F}-\{\mathrm{e}\}$ is not an edge cover of $\mathrm{G}$.

Definition 2.4 [3] An edge cover with minimum cardinality is a minimum edge cover of the graph G. The cardinality of a minimum edge cover is called the edge covering number of $G$ and it is denoted by $\alpha_{1}(\mathrm{G})$.

For an edge set $F$ of the graph $G, V(F)$ denotes the set of end vertices of edges of $F$.

Note that the graph with isolated vertices does not admit an edge cover. Therefore in case of edge cover problems, we will consider only those graphs which do not contain isolated vertices.

\section{VERTEX REMOVAL FROM THE GRAPH}

In subsequent results, we will assume that $\mathrm{G}-\mathrm{v}$ does not have isolated vertices. We prove that the edge covering number of the graph does not increase when a vertex is removed from the graph.

Theorem 3.1 Let $\mathrm{G}$ be a graph and $\mathrm{v} \in \mathrm{V}(\mathrm{G})$ then $\alpha_{1}(\mathrm{G}-\mathrm{v}) \leq \alpha_{1}(\mathrm{G})$.

Proof. Let $\mathrm{G}$ be a graph and $\mathrm{v} \in \mathrm{V}(\mathrm{G})$. Let $\mathrm{F}$ be a minimum edge cover of $\mathrm{G}$ and suppose that $\mathrm{vu}_{1}, \mathrm{vu}_{2}, \ldots, \mathrm{vu}_{\mathrm{k}}$ are all the edges incident at $\mathrm{v}$ such that $\left\{\mathrm{vu}_{1}, \mathrm{vu}_{2}, \ldots, \mathrm{vu}_{\mathrm{k}}\right\} \subseteq \mathrm{F}$. Now consider the subgraph $\mathrm{G}-\mathrm{v}$. Let $\mathrm{m}$ be the number of edges of $\mathrm{G}-\mathrm{v}$ which are incident with atleast one vertex of $u_{1}, u_{2}, \ldots, u_{k}$ but they are not members of $F$. Let these edges be $f_{1}, f_{2}, \ldots$, $\mathrm{f}_{\mathrm{m}}$. Now consider the set $\mathrm{F}_{1}=\left(\mathrm{F}-\left\{\mathrm{vu}_{1}, \mathrm{vu}_{2}, \ldots, \mathrm{vu}_{\mathrm{k}}\right\}\right) \cup\left\{\mathrm{f}_{1}, \mathrm{f}_{2}, \ldots, \mathrm{f}_{\mathrm{m}}\right\}$ then obviously $\mathrm{F}_{1}$ is an edge cover of $\mathrm{G}-\mathrm{v}$ and $\left|\mathrm{F}_{1}\right| \leq|\mathrm{F}|$. Thus, $\alpha_{1}(\mathrm{G}-\mathrm{v}) \leq \alpha_{1}(\mathrm{G})$.

From the proof of theorem 3.1, we have the following corollary.

Corollary 3.2 Let $\mathrm{G}$ be a graph and $\mathrm{v} \in \mathrm{V}(\mathrm{G})$ then $\alpha_{1}(\mathrm{G}-\mathrm{v})<\alpha_{1}(\mathrm{G})$ if $\mathrm{m}<\mathrm{k}$.

Theorem 3.3 Let $\mathrm{G}$ be a graph and $\mathrm{v} \in \mathrm{V}(\mathrm{G})$ then $\alpha_{1}(\mathrm{G}-\mathrm{v})<\alpha_{1}(\mathrm{G})$ if and only if there is a minimum edge cover $F$ of $G$ such that whenever $u v \in F$, there is an edge uw of $G$ such that $u w$ $\in \mathrm{F}$.

Proof. Suppose the condition is satisfied. Let $F$ be the minimum edge cover of $G$ which satisfies the requirement. Since $F$ is an edge cover of $G$, there is some edge uv such that $u v \in F$. By the assumption, there is an edge $u w$ such that $u w \in F$. Consider the set $F_{1}=F-\{u v\}$. Since uw $\in$ $\mathrm{F}_{1}, \mathrm{~F}_{1}$ is an edge cover of $\mathrm{G}-\mathrm{v}$. Therefore $\alpha_{1}(\mathrm{G}-\mathrm{v}) \leq\left|\mathrm{F}_{1}\right|<|\mathrm{F}|=\alpha_{1}(\mathrm{G})$.

Conversely, Suppose that $\alpha_{1}(\mathrm{G}-\mathrm{v})<\alpha_{1}(\mathrm{G})$. Let $\mathrm{F}_{1}$ be a minimum edge cover of $\mathrm{G}-\mathrm{v}$ then $\mathrm{F}_{1}$ cannot be an edge cover of $\mathrm{G}$. Therefore there is no edge containing $\mathrm{v}$ which is a member of $\mathrm{F}_{1}$. Let $\mathrm{uv}$ be any edge containing $\mathrm{v}$ and let $\mathrm{F}=\mathrm{F}_{1} \cup\{\mathrm{uv}\}$ then obviously $\mathrm{F}$ is an edge cover of $\mathrm{G}$. Also since $\alpha_{1}(\mathrm{G}-\mathrm{v})<\alpha_{1}(\mathrm{G}), \mathrm{F}$ is a minimum edge cover of $\mathrm{G}$ and $\mathrm{F}$ contains the edge uv. Since $\mathrm{u}$ is a vertex of $\mathrm{G}-\mathrm{v}$ there is an edge $u w$ in $F_{1}$ then uw is also in $F$. Thus $F$ satisfies the requirement.

Corollary 3.4 If $\alpha_{1}(\mathrm{G}-\mathrm{v})<\alpha_{1}(\mathrm{G})$ then there is a minimum edge cover $\mathrm{F}$ of $\mathrm{G}$ containing a unique edge uv containing $\mathrm{v}$ such that for some edge uw, uw $\in \mathrm{F}$.

Proof. Since $\alpha_{1}(\mathrm{G}-\mathrm{v})<\alpha_{1}(\mathrm{G})$. There is a minimum edge cover $\mathrm{F}$ of $\mathrm{G}$ such that whenever uv $\in F$, there is an edge uw which is in $F$. Now $F$ is a minimal edge cover of $G$ and therefore by theorem 1.3, one of the following two conditions hold. 
(1) All the edges incident at $\mathrm{u}$ (except uv) are outside F.

(2) All the edges incident at $v$ (except uv) are outside F.

Since (1) does not hold, (2) must hold. Therefore any edge containing $\mathrm{v}$ other than uv must be outside $\mathrm{F}$. Thus uv is the unique edge containing $\mathrm{v}$ which is in $\mathrm{F}$.

\section{Counting the number of minimum edge covers of the graph}

Remark 3.5 Suppose that $\alpha_{1}(\mathrm{G}-\mathrm{v})<\alpha_{1}(\mathrm{G})$. Let $\mathrm{F}_{1}$ be a minimum edge cover of $\mathrm{G}-\mathrm{v}$ then by theorem 3.3, $\mathrm{F}_{1} \cup\{\mathrm{uv}\}$ is a minimum edge cover of $\mathrm{G}$. Thus, every minimum edge cover of $\mathrm{G}-\mathrm{v}$ gives rise to $\operatorname{deg}(\mathrm{v})$ number of minimum edge covers of $\mathrm{G}$. Suppose there are $\mathrm{m}$ minimum edge covers of $\mathrm{G}-\mathrm{v}$ then they will give rise to $\mathrm{m} * \operatorname{deg}(\mathrm{v})$ number of minimum edge covers of $\mathrm{G}$ (containing v) with possible repetition.

Let $v_{1}, v_{2}, \ldots, v_{k}$ be the vertices of $G$ such that for $i=1,2,3, \ldots, k$,

(1) $G-v_{i}$ does not have isolated vertices.

(2) $\alpha_{1}\left(\mathrm{G}-\mathrm{v}_{\mathrm{i}}\right)<\alpha_{1}(\mathrm{G})$ then there are atmost

$$
\sum_{\mathrm{i}=1}^{k} \mathrm{~m}_{\mathrm{i}} * \operatorname{deg}\left(\mathrm{v}_{\mathrm{i}}\right)
$$

minimum edge covers of $G$ corresponding to vertices $v_{i}$ with possible repetition, where $m_{i}$ denotes the number of minimum edge covers of $\mathrm{G}-\mathrm{v}_{\mathrm{i}}(\mathrm{i}=1,2,3, \ldots, \mathrm{k})$.

Example 3.6 Consider the cycle graph $\mathrm{G}=\mathrm{C}_{5}$ with vertices $1,2,3,4,5$.

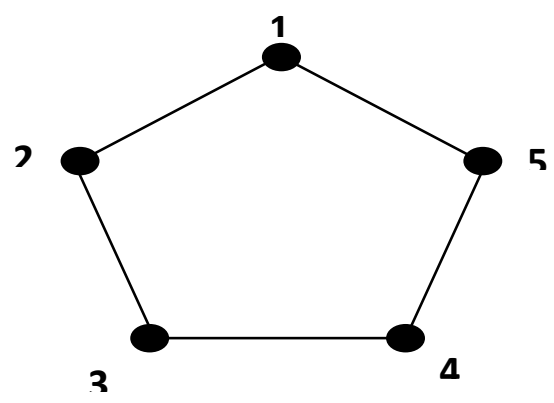

Figure 1. $\mathbf{G}=\mathbf{C}_{5}$

Note that $\alpha_{1}\left(\mathrm{G}-\mathrm{v}_{\mathrm{i}}\right)<\alpha_{1}(\mathrm{G})$ for all vertices $\mathrm{v}_{\mathrm{i}}=1,2,3,4,5$. The graph $\mathrm{G}-\mathrm{v}_{\mathrm{i}}$ has only one minimum edge cover (for any $\left.v_{i}=1,2,3,4,5\right)$ also $\operatorname{deg}\left(v_{i}\right)=2$ for each $v_{i}=1,2,3,4,5$. Therefore the graph $\mathrm{G}$ has atmost

$$
\sum_{\mathrm{i}=1}^{k} \mathrm{~m}_{\mathrm{i}} * \operatorname{deg}\left(\mathrm{v}_{\mathrm{i}}\right)=(1 * 2)+(1 * 2)+(1 * 2)+(1 * 2)+(1 * 2)=10
$$

edge covers with possible repetition. The edge covers of the graph $\mathrm{G}$ are obtained as follows.

For $v_{i}=5, F_{1}=\{12,34\}$ is the only one minimum edge cover of $G-5$. Since $\operatorname{deg}(5)=2$ and the vertices 1 and 4 are neighbors of 5 . The minimum edge covers of $G$ correspond to vertex 5 are $E_{5}^{1}=\{12,34\} \cup\{51\}$ and $E_{5}^{2}=\{12,34\} \cup\{54\}$. Similarly, the minimum edge covers of $G$ correspond to vertices $4,3,2,1$ are $E_{4}^{1}=\{15,23\} \cup\{45\}$ and $E_{4}^{2}=\{15,23\} \cup\{43\}, E_{3}^{1}=\{45$, $12\} \cup\{34\}$ and $E_{3}^{2}=\{45,12\} \cup\{32\}, E_{2}^{1}=\{34,15\} \cup\{23\}$ and $E_{2}^{2}=\{34,15\} \cup\{21\}, E_{1}^{1}=$ $\{23,45\} \cup\{15\}$ and $E_{1}^{2}=\{23,45\} \cup\{12\}$ respectively.

Remark 3.7 From the above discussion, it is clear that if $\alpha_{1}(\mathrm{G}-\mathrm{v})<\alpha_{1}(\mathrm{G})$ then the number of minimum edge covers of the graph $\mathrm{G}$ is greater than the number of minimum edge covers of $\mathrm{G}-\mathrm{v}$.

\section{VERTEX COVER IN HYPER GRAPH}

Definition 4.1 [1] The hypergraph is an order pair $G=(V, E)$. The nonempty set $V$ contains the elements $\left\{\mathrm{v}_{1}, \mathrm{v}_{2}, \ldots, \mathrm{v}_{\mathrm{n}}\right\}$ and $\mathrm{E}=\left\{\mathrm{C}_{1}, \mathrm{C}_{2}, \ldots, \mathrm{C}_{\mathrm{m}}\right\}$ is a family of subsets of $\mathrm{V}$ such that 


$$
\bigcup_{i=1}^{m} C_{i}=V
$$

each $\mathrm{Ci}$ is nonempty. The elements of $\mathrm{V}$ and $\mathrm{E}$ are called the vertices and the edges of the hypergraph $\mathrm{G}$ respectively.

We make the following conventions about the hypergraph considered in this paper.

(1) If $\mathrm{x}$ and $\mathrm{y}$ are distinct vertices then there is atmost one edge which contains $\mathrm{x}$ and $\mathrm{y}$.

(2) Any two distinct edges of the hypergraph will intersects in atmost one vertex.

(3) If $\mathrm{e}$ and $\mathrm{f}$ are edges having atleast two vertices then $\mathrm{e} \nsubseteq \mathrm{f}$ and $\mathrm{f} \nsubseteq \mathrm{e}$.

Definition 4.2 [1] Let $G=(V, E)$ be a hypergraph and let $v \in V(G)$ be a vertex of $G$. A sub hypergraph $G-v$ is a hypergraph whose vertex set is $V-\{v\}$ and the edge set is equal to $\left\{\mathrm{e}^{\prime} \mid \mathrm{e}^{\prime}\right.$ is non empty and $\mathrm{e}^{\prime}=\mathrm{e}-\{\mathrm{v}\}$, e $\left.\in \mathrm{E}(\mathrm{G})\right\}$.

Definition 4.3 [1] Let $G=(V, E)$ be a hypergraph and let $v \in V(G)$ be a vertex of $G$. A partial sub hypergraph of $G$ is a hypergraph with vertex set $V-\{v\}$ and the edge set equal to $\{e \in E(G) \mid$ $\mathrm{v} \notin \mathrm{e}\}$.

If $G$ is a graph then $G$ can also be regarded as a hypergraph. Therefore the dual hypergraph $G^{*}$ of the graph $G$ is defined as follows.

Definition 4.4 [1] Let $G=(V(G), E(G))$ be a graph. The dual hypergraph $G^{*}$ of the graph $G$ is a hypergraph with vertex set $V\left(G^{*}\right)=E(G)$ and edge set $E\left(G^{*}\right)=\{\bar{v} \mid v \in V(G)\}$ where $\bar{v}=\{e \in$ $E(G) \mid v$ is an end vertex of e\}.

Definition 4.5 A set of vertices $S$ of the hypergraph $G$ is a vertex cover of $G$ if every edge of the hypergraph $G$ has nonempty intersection with the set $S$. That is, for every e $\in E(G)$, e $\cap S \neq \emptyset$.

The cardinality of a minimum vertex cover of the hypergraph $\mathrm{G}$ is the vertex covering number of $\mathrm{G}$, denoted by $\alpha_{0}(\mathrm{G})$.

It is clear that any edge cover of the graph $G$ is a vertex cover of the dual hypergraph $G^{*}$.

Example 4.6 Consider the graph $\mathrm{G}=\mathrm{C}_{4}$ with vertices $1,2,3$, 4. The edges of $\mathrm{C}_{4}$ are 12, 23, 34, 41 .

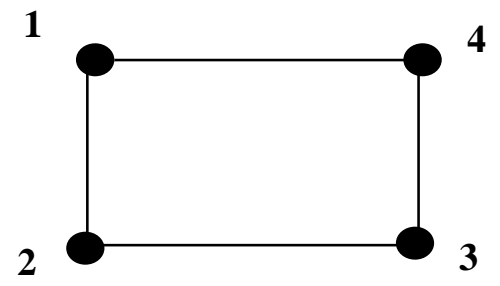

Figure 2. $\mathrm{G}=\mathrm{C}_{4}$

The edge set $\{12,34\}$ is an edge cover of the graph $G$. The vertex set of the dual hypergraph $G^{*}$ is $V\left(G^{*}\right)=\{12,23,34,41\}$ and the edges are $\overline{1}=\{12,41\}, \overline{2}=\{12,23\}, \overline{3}=\{23,34\}$ and $\overline{4}=\{34$, $41\}$. Thus $\{12,34\}$ is a vertex cover of the dual hypergraph $G^{*}$.

\section{Vertex Removal from the Hypergraph}

We observe the effect of vertex removal on the vertex covering number of hypergraph. In the following theorems, we consider the sub hypergraph $\mathrm{G}-\mathrm{v}$ obtained by removing a vertex $\mathrm{v}$ from the hypergraph $\mathrm{G}$.

Theorem 4.7 [4] Let $G$ be a hypergraph and $v \in V(G)$ such that $\{v\}$ is not an edge of $G$ then $\alpha_{0}(\mathrm{G}-\mathrm{v}) \geq \alpha_{0}(\mathrm{G})$.

Proof. Let $S$ be a minimum vertex cover of $G-v$ and e be an edge of $G$. Let $e^{\prime}=e-\{v\}$. Since $S$ is a vertex cover of $G-v, e^{\prime} \cap S \neq \emptyset$. Therefore $e \cap S \neq \varnothing$. Thus $S$ is a vertex cover of $G$. Thus $\alpha_{0}(\mathrm{G}) \leq|\mathrm{S}|=\alpha_{0}(\mathrm{G}-\mathrm{v})$. 
Theorem 4.8 [4] Let $\mathrm{G}$ be a hypergraph and $\mathrm{v} \in \mathrm{V}(\mathrm{G})$ such that $\{\mathrm{v}\}$ is not an edge of $\mathrm{G} . \alpha_{0}(\mathrm{G}-$ $\mathrm{v})=\alpha_{0}(\mathrm{G})$ if and only if there is a minimum vertex cover $\mathrm{S}$ of $\mathrm{G}$ such that $\mathrm{v} \notin \mathrm{S}$.

Proof. Let $S$ be a minimum vertex cover of $G$ such that $v \notin S$ then $S$ is a vertex set of the sub hypergraph $G-v$. Let $e^{\prime}$ be any edge of $G-v$ and $e$ be the edge of $G$ such that $e-\{v\}=e^{\prime}$. Now $\mathrm{e} \cap \mathrm{S} \neq \varnothing$ and since $\mathrm{v} \notin S$, e $\cap \mathrm{S} \neq \varnothing$. Thus $S$ is a vertex cover of the sub hypergraph $\mathrm{G}-$ v. Therefore $\alpha_{0}(\mathrm{G}-\mathrm{v}) \leq|\mathrm{S}|=\alpha_{0}(\mathrm{G}) \leq \alpha_{0}(\mathrm{G}-\mathrm{v})$. Thus $\alpha_{0}(\mathrm{G}-\mathrm{v})=\alpha_{0}(\mathrm{G})$.

Conversely, $\alpha_{0}(\mathrm{G}-\mathrm{v})=\alpha_{0}(\mathrm{G})$. Let $\mathrm{S}$ be a minimum vertex cover of $\mathrm{G}-\mathrm{v}$ then obviously $\mathrm{S}$ is a vertex cover of $\mathrm{G}$ also. Since $\alpha_{0}(\mathrm{G}-\mathrm{v})=\alpha_{0}(\mathrm{G}), \mathrm{S}$ is a minimum vertex cover of $\mathrm{G}$ not containing $\mathrm{v}$.

Corollary 4.9 [4] Let $\mathrm{G}$ be a hypergraph and $\mathrm{v} \in \mathrm{V}(\mathrm{G}) . \alpha_{0}(\mathrm{G}-\mathrm{v})>\alpha_{0}(\mathrm{G})$ if and only if for every minimum vertex cover $\mathrm{S}$ of $\mathrm{G}, \mathrm{v} \in \mathrm{S}$.

\section{EDGE REMOVAL FROM THE GRAPH}

We consider the operation edge removal from the graph. We assume that when a particular edge e is removed from the graph $\mathrm{G}$ then $\mathrm{G}-\mathrm{e}$ does not have isolated vertices.

The effect of edge removal on the edge covering number of the graph is indicated with the examples (i) and (ii) of figure 3.

Consider the graphs of row 2 in the following figure 3 . The graphs after removal of an edge from each graph of row 2 are given in row 1.

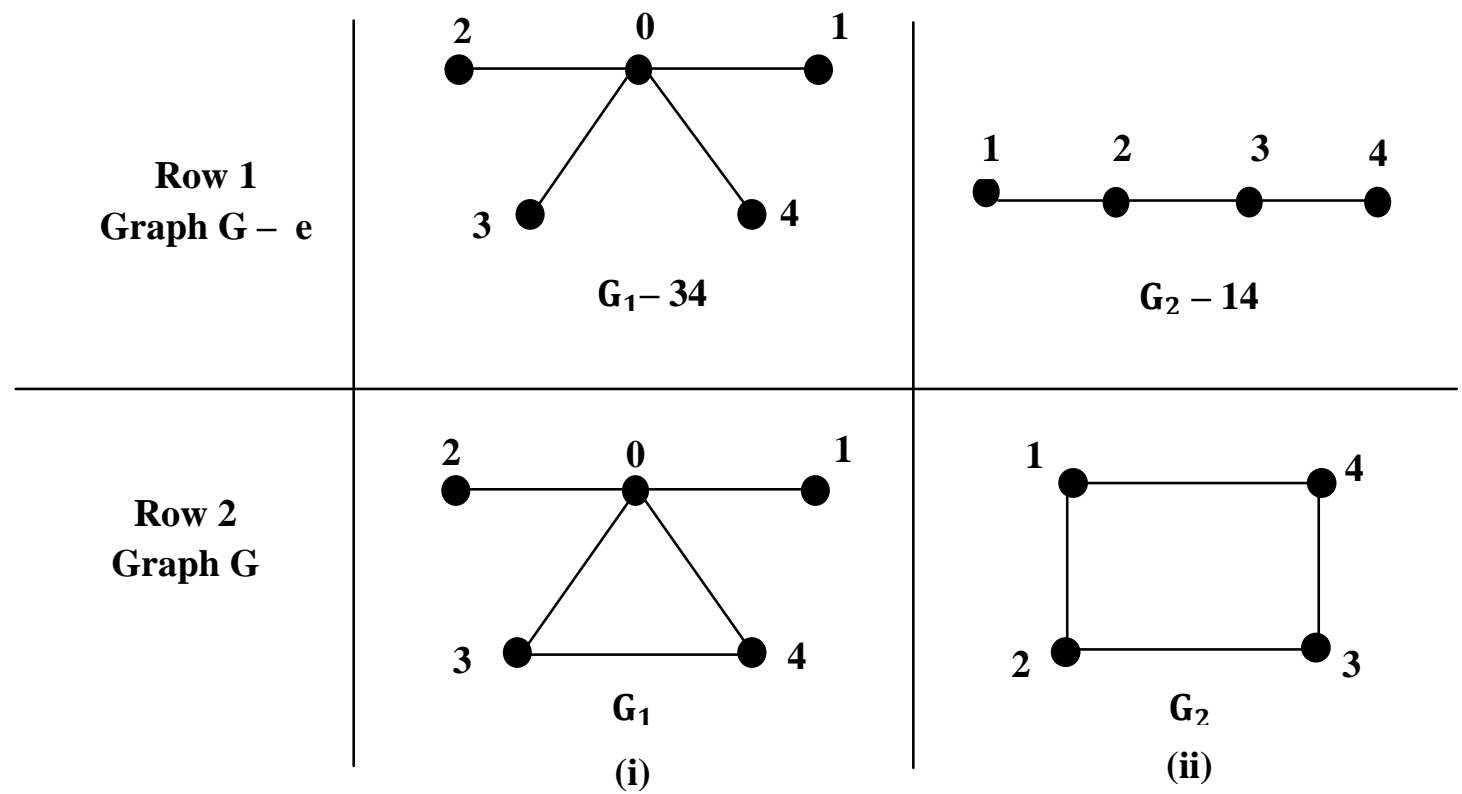

Figure 3

(i) Consider the graph $G_{1}$ of Figure 3 ((i) of Row 2). The minimum edge cover of this graph is $\{01$, $02,34\}$. Therefore the edge covering number of the graph is 3 . By removing an edge 34 from the graph, the edge covering number of the resultant star graph $\mathrm{G}_{1}-34$ ((i) of Row 1) is 4. Thus, the edge covering number of the graph increases when an edge is removed from the graph.

(ii) Consider the graph $G_{2}$ ((ii) of Row 2). The minimum edge covering sets of $G_{2}$ are $\{12,34\}$, $\{23,41\}$. The edge covering number of $G_{2}$ is 2 . If any edge is removed from $G_{2}$ then it gives a path graph $\mathrm{P}_{4}$ ((ii) of Row 1). The edge covering number of $\mathrm{P}_{4}$ is also 2 . In this example, the edge covering number does not change when an edge is removed from the graph.

Theorem 5.1 Let e be an edge of the graph $G$ such that $G-e$ does not have any isolated vertex then $\alpha_{1}(\mathrm{G}-\mathrm{e}) \geq \alpha_{1}(\mathrm{G})$.

Proof. Let $F$ be an edge cover of $G-e$. Since $G-e$ and $G$ have the same vertex set, $F$ is also an edge cover of $\mathrm{G}$. Thus $\alpha_{1}(\mathrm{G}-\mathrm{e})=|\mathrm{F}| \geq \alpha_{1}(\mathrm{G})$. 
Another proof of the theorem 5.1 Suppose that $G$ is a graph. Consider the dual

hypergraph $G^{*}$ of $G$. Let $e=x y$ then $\bar{x}=\{f \in E(G) \mid x \in f\}$ and $\bar{y}=\{f \in E(G) \mid y \in f\}$.

Now consider the dual hypergraph $(G-e)^{*}$ of $G-e$. In the dual hypergraph $(G-e)^{*}, \bar{x}$ will not contain e and $\bar{y}$ will not contain e. All other edges of $G^{*}$ will be the edges of $(G-e)^{*}$ and all other edges will remain, that is, $\bar{z}^{\prime}=\bar{z}-\{e\}=\bar{z}$ for all $\mathrm{z} \notin\{x, y\}$ and $\bar{x}^{\prime}=\bar{x}-\{e\}, \bar{y}^{\prime}=\bar{y}-\{e\}$. Thus, the dual hypergraph $(\mathrm{G}-\mathrm{e})^{*}$ is the sub hypergraph $\mathrm{G}^{*}-\mathrm{e}$. Therefor by theorem $4.1, \alpha_{1}(\mathrm{G}-$ e) $\geq \alpha_{1}(\mathrm{G})$.

Theorem 5.2 Let $\mathrm{G}$ be a graph and $\mathrm{e} \in \mathrm{E}(\mathrm{G})$ such that $\mathrm{G}-\mathrm{e}$ does not have any isolated vertex then $\alpha_{1}(\mathrm{G}-\mathrm{e})=\alpha_{1}(\mathrm{G})$ if and only if there is a minimum edge cover $\mathrm{F}$ of $\mathrm{G}$ such that $\mathrm{e}=\mathrm{xy} \notin \mathrm{F}$.

Proof. Suppose that the condition is satisfied then obviously $F$ is an edge cover of $G-e$. Therefore $\alpha_{1}(\mathrm{G}-\mathrm{e}) \leq|\mathrm{F}|=\alpha_{1}(\mathrm{G}) \leq \alpha_{1}(\mathrm{G}-\mathrm{e})$. Thus $\alpha_{1}(\mathrm{G}-\mathrm{e})=\alpha_{1}(\mathrm{G})$.

Suppose that $\alpha_{1}(\mathrm{G}-\mathrm{e})=\alpha_{1}(\mathrm{G})$. Let $\mathrm{F}$ be a minimum edge cover of $\mathrm{G}-\mathrm{e}$. If $\mathrm{e}=\mathrm{xy}$ then there is an edge $h$ of $G-e$ incident at $x$ such that $h \in F$. Similarly, there is an edge $h^{\prime}$ of $G-e$ incident at $y$ such that $h^{\prime} \in F$. Thus $F$ is an edge cover of $G$ with $e \notin F$.

Corollary 5.3 Let e be an edge of the graph $G$ such that $G-e$ does not have any isolated vertex then $\alpha_{1}(\mathrm{G}-\mathrm{e})>\alpha_{1}(\mathrm{G})$ if and only if for every minimum edge cover $\mathrm{F}$ of $\mathrm{G}, \mathrm{e}=\mathrm{xy} \in \mathrm{F}$.

Proof. Suppose that $\alpha_{1}(\mathrm{G}-\mathrm{e})>\alpha_{1}(\mathrm{G})$.

Consider the dual hypergraph $G^{*}$ of the graph $G$ then $e$ is a vertex of the dual hypergraph $G^{*}$. Obviously, $\alpha_{0}\left(\mathrm{G}^{*}-\mathrm{e}\right)>\alpha_{0}\left(\mathrm{G}^{*}\right)$. Therefore by corollary 4.3 , for every minimum vertex cover $\mathrm{F}$ of the dual hypergraph $G^{*}$, e $\in F$. This is equivalent to say that $e \in F$ for every minimum edge cover $F$ of the graph $\mathrm{G}$.

Conversely, suppose that $e \in F$ for every minimum edge cover $F$ of the graph $G$ then $e \in F$ for every minimum vertex cover $\mathrm{F}$ of the dual hypergraph $\mathrm{G}^{*}$. Therefore again by corollary $4.3, \alpha_{0}\left(\mathrm{G}^{*}-\right.$ e) $>\alpha_{0}\left(\mathrm{G}^{*}\right)$. It is equivalent to $\alpha_{1}(\mathrm{G}-\mathrm{e})>\alpha_{1}(\mathrm{G})$.

\section{Edge AdDition to THE GraPH}

We add a single edge between two non-adjacent vertices of the graph. The operation is known as edge addition to the graph. We observe the effect on the edge covering number of the graph after adding a new edge. If we consider the graphs of row 1 (figure 3 ) as $G$ and the graphs of row 2 (figure 3) as $G$ $+e$ then from (i) and (ii) of figure 3.

(i) The minimum edge cover of the star graph ((i) of Row 1) is $\{01,02,03,04\}$. Therefore the edge covering number of the star graph is 4 . By adding an edge 34 between the non-adjacent vertices 3 and 4 then the edge covering number of the resultant graph $G_{1}$ ((i) of Row 2) is 3. Thus, the edge covering number of the graph decreases when an edge is added between two non-adjacent vertices of the graph.

(ii) The minimum edge cover of path graph ((ii) of Row 1) is $\{12,34\}$. Therefore the edge covering number is 2 . If an extra edge is added between the vertices 1 and 4 of the path graph then it gives the graph $G_{2}$ ((ii) of Row 2). The edge covering number of $G_{2}$ is also 2. In this example, the edge covering number does not change when an edge is added between two non-adjacent vertices of the graph $G_{1}$.

Theorem 6.1 Let $\mathrm{G}$ be a graph. $\mathrm{x}$ and $\mathrm{y}$ are two non-adjacent vertices of the graph $\mathrm{G}$ then $\alpha_{1}(\mathrm{G}$ $+\mathrm{xy}) \leq \alpha_{1}(\mathrm{G})$.

Proof. Let $F$ be a minimum edge cover of the graph $G$ then obviously $F$ is an edge cover of $\mathrm{G}+$ xy. Thus, $\alpha_{1}(\mathrm{G}+\mathrm{xy}) \leq|\mathrm{F}|=\alpha_{1}(\mathrm{G})$. Hence, $\alpha_{1}(\mathrm{G}+\mathrm{xy}) \leq \alpha_{1}(\mathrm{G})$.

Theorem 6.2 Let $\mathrm{G}$ be a graph without isolated vertex. $\mathrm{x}$ and $\mathrm{y}$ are two non-adjacent vertices of the graph G. $\alpha_{1}(\mathrm{G}+\mathrm{xy})<\alpha_{1}(\mathrm{G})$ if and only if there is a minimum edge cover $\mathrm{F}$ of $\mathrm{G}$ such that the following conditions are satisfied.

(1) There is an edge $x x^{\prime}$ in $F$. 
(2) There is an edge yy' in F.

(3) There is an edge $f$ containing $x^{\prime}$ such that $f \neq x x^{\prime}$ and $f \in F$.

(4) There is an edge $h$ containing $y^{\prime}$ such that $h \neq y y^{\prime}$ and $h \in F$.

Proof. Suppose that the conditions are satisfied.

Consider the set $\mathrm{F}_{1}=(\mathrm{F} \cup\{\mathrm{xy}\})-\left\{\mathrm{xx}^{\prime}, \mathrm{yy}^{\prime}\right\}$ then $\left|\mathrm{F}_{l}\right|<|\mathrm{F}|$. Let $\mathrm{z}$ be any vertex of $\mathrm{G}+\mathrm{xy}$. If $\mathrm{z}$ $=x^{\prime}$ then the edge $f$ contains the vertex $z$ and $f \in F_{l}$. Similarly if $z=y^{\prime}$ then the edge $h$ contains the vertex $z$ and $h \in F_{1}$. If $z=x$ or $z=y$ then the edge $x y$ contains $z$ and $x y \in F_{1}$.

Suppose that $z \notin\left\{x, x^{\prime}, y, y^{\prime}\right\}$. Since $F$ is an edge cover of $G$, there is an edge $g$ of $G$ containing $\mathrm{z}$ such that $\mathrm{g} \in \mathrm{F}$. Note that $\mathrm{g} \neq \mathrm{xx}^{\prime}, \mathrm{g} \neq \mathrm{y} \mathrm{y}^{\prime}$ and also $\mathrm{g} \neq \mathrm{xy}$. Therefore $\mathrm{g} \in \mathrm{F}_{1}$. Therefore $g$ is an edge of $G+x y$ such that $g \in F_{1}$. Thus $F_{1}$ is an edge cover of $G+x y$. Thus $\alpha_{1}(\mathrm{G}+\mathrm{xy})<\alpha_{1}(\mathrm{G})$.

Conversely, suppose that $\alpha_{1}(\mathrm{G}+\mathrm{xy})<\alpha_{1}(\mathrm{G})$. Let $\mathrm{F}_{1}$ be a minimum edge cover of $\mathrm{G}+\mathrm{xy}$. If $\mathrm{xy}$ $\notin \mathrm{F}_{1}$ then $\mathrm{F}_{1}$ is an edge cover of $\mathrm{G}$. Therefore, $\alpha_{1}(\mathrm{G}+\mathrm{xy}) \leq \alpha_{1}(\mathrm{G})$. Which is a contradiction. Therefore $x y \in F_{1}$. Now let $F=F_{1}-\{x y\}$ then $F$ cannot be an edge cover of $G+x y$ because $|F|<\left|F_{1}\right|$. Therefore $x y$ is the only edge of $F_{1}$ which contains $x$ and $x y$ is the only edge of $F_{1}$ which contains $y$. Let $\mathrm{xx}^{\prime}$ be any edge of $\mathrm{G}$ such that $\mathrm{xx}^{\prime} \neq \mathrm{xy}$ and $\mathrm{yy}^{\prime}$ be any edge of $\mathrm{G}$ such that $y y^{\prime} \neq x y$. Now let $H=F_{1} \cup\left\{x x^{\prime}\right.$, yy' $\}$ then $|H|>\left|F_{1}\right|$. Now $x^{\prime}$ is a vertex of $G+x y$ and $F_{1}$ is an edge cover of $G+x y$ therefore there is an edge $f$ of $F_{1}$ which contains $x^{\prime}$. Note that $f \neq$ $\mathrm{xx}^{\prime}$ because $\mathrm{xx}^{\prime} \notin \mathrm{F}_{1}$. Similarly there is an edge $\mathrm{h}$ containing $\mathrm{y}^{\prime}$ such that $\mathrm{h} \in \mathrm{F}_{1}$ and $\mathrm{h} \neq \mathrm{yy} \mathrm{y}^{\prime}$ because yy' $\notin \mathrm{F}_{1}$. Obviously $\mathrm{f}, \mathrm{h} \in \mathrm{H}$.

Let $\mathrm{z}$ be any vertex of $\mathrm{G}$. If $\mathrm{z}=\mathrm{x}$ then $\mathrm{xx}^{\prime}$ contains $\mathrm{z}$. Similarly if $\mathrm{z}=\mathrm{y}$ then $\mathrm{yy}$ ' contains $\mathrm{z}$. Suppose that $z \notin\{x, y\}$. Now $z$ is a vertex of $G+x y$. Since $F_{1}$ is an edge cover of $G+x y$, there is an edge $g$ of $\mathrm{G}+\mathrm{xy}$ which contains $\mathrm{z}$. Obviously $\mathrm{g} \neq \mathrm{xy}$ therefore $\mathrm{g}$ is an edge of $\mathrm{H}$. Thus $\mathrm{H}$ is an edge cover of $\mathrm{G}$ and satisfies the required conditions.

\section{REFERENCES}

[1] C. Berge, Graphs and Hypergraphs, Amsterdam, 1973.

[2] F. Harary, Graph Theory, Addison-Wesley, Reading Mass., 1969.

[3] T. W. Haynes, S. T. Hedetniemi and P. J. Slater, Fundamental of Domination in Graphs, Marcel Dekker, Inc., New York, 1998.

[4] D. K. Thakkar, K. N. Kalariya, Vertex Covering and Stability in Hypergraphs, IJCAM, 11(1)(2016), $61-69$. 